\title{
Effects of Chitosan Alkali Pretreatment on the Preparation of Electrospun PCL/Chitosan Blend Nanofibrous Scaffolds for Tissue Engineering Application
}

\author{
Fatemeh Roozbahani, ${ }^{1}$ Naznin Sultana, ${ }^{1}$ Ahmad Fauzi Ismail, ${ }^{2}$ and Hamed Nouparvar ${ }^{3}$ \\ ${ }^{1}$ Faculty of Biosciences and Medical Engineering, Universiti Teknologi Malaysia (UTM), 81310 Skudai, Johor Bahru, Johor, Malaysia \\ ${ }^{2}$ Advanced Membrane Technology Center, Universiti Teknologi Malaysia (UTM), 81310 Johor Bahru, Johor, Malaysia \\ ${ }^{3}$ Faculty of Chemical Engineering, Department of Polymer, Universiti Teknologi Malaysia (UTM), 81310 Johor Bahru, Johor, Malaysia \\ Correspondence should be addressed to Naznin Sultana; naznin@biomedical.utm.my
}

Received 19 March 2013; Revised 12 July 2013; Accepted 23 July 2013

Academic Editor: Jin Zhang

Copyright (C) 2013 Fatemeh Roozbahani et al. This is an open access article distributed under the Creative Commons Attribution License, which permits unrestricted use, distribution, and reproduction in any medium, provided the original work is properly cited.

Recently, nanofibrous scaffolds have been used in the field of biomedical engineering as wound dressings, tissue engineering scaffolds, and drug delivery applications. The electrospun nanofibrous scaffolds can be used as carriers for several types of drugs, genes, and growth factors. PCL is one of the most commonly applied synthetic polymers for medical use because of its biocompatibility and slow biodegradability. PCL is hydrophobic and has no cell recognition sites on its structure. Electrospinning of chitosan and PCL blend was investigated in formic acid/acetic acid as the solvent with different PCL/chitosan ratios. High viscosity of chitosan solutions makes difficulties in the electrospinning process. Strong hydrogen bonds in a 3D network in acidic condition prevent the movement of polymeric chains exposed to the electrical field. Consequently, the amount of chitosan in PCL/chitosan blend was limited and more challenging when the concentration of PCL increases. The treatment of chitosan in alkali condition under high temperature reduced its molecular weight. Longer treatment time further decreased the molecular weight of chitosan and hence its viscosity. Electrospinning of PCL/chitosan blend was possible at higher chitosan ratio, and SEM images showed a decrease in fiber diameter and narrower distribution with increase in the chitosan ratio.

\section{Introduction}

Electrospinning has received widespread attention as an easy and adaptable technique which leads to fabrication of fibers in nanometer size scale. Nanofibers provide remarkably high surface area for surface interchanges, a significantly improved interconnected pore architecture for diffusion and transportation of biological fluid, nutrient, and drugs for tissue engineering and drug delivery applications [1]. Utilizing electrospun fibers and nanofibrous meshes in tissue engineering applications often includes several considerations: selection of material, fiber orientation (aligned or random), porosity, and surface modification. Both natural and synthetic (biodegradable and nondegradable) materials as well as hybrid blends can be used depending on specified mechanical and biomimetic properties $[2,3]$. Also by varying the processing and solution parameters, fiber orientation and porosity/pore size of the electrospun scaffold can be controlled and optimized for each individual application.

In tissue engineering, the scaffold prepares a threedimensional (3D) construct for cell attachment, proliferation, and formation of an extracellular matrix (ECM), as well as a carrier of growth factors or other biomolecular signals [4]. Scaffolds for tissue engineering should have good mechanical properties, suitable biodegradability, and most importantly good biocompatibility $[4,5]$. Particularly, the surface properties of the material define the interactions between the cells and the material and, consequently, affect cell adhesion [6].

Poly( $\varepsilon$-caprolactone) (PCL) is one of the most commonly applied synthetic polymers for medical use because of its biocompatibility and slow biodegradability. PCL is hydrophobic, so presence of another component which embeds hydrophilicity and cell attachment is necessary in scaffold fabrication. Electrospinning of pure PCL has been 
studied expansively [7-13]. Van der Schueren et al. [13] introduced an acetic acid/formic acid (AA/FA) solvent system for the electrospinning of PCL. Chitosan has been widely used in different biomedical areas because of its various advantages like biocompatibility, biodegradability, hydrophilicity, nonantigenicity, antimicrobial activity, nontoxicity, bioadherence, and cell affinity, which make it the ideal candidate for uses in a wide range of applications. It is a linear biopolymer and is usually obtained by alkaline deacetylation of chitin, the second most abundant biopolymer in nature after cellulose [14]. Chitosan is soluble in organic acids, such as aqueous acetic acid and formic acid. Free amino groups alongside chitosan chain become positively charged in acidic condition. Formation of hydrogen bonds in 3D network inhibits chain movement and limits the electrospinning. This drawback is a challenge for researchers and they have come up with different solutions which can be put in two categories: firstly using a suitable solvent system and secondly blending chitosan with another polymer. Ohkawa et al. [15] successfully prepared pure chitosan nanofibers using trifluoroacetic acid (TFA) as solvent. TFA can form salts with the amino groups of chitosan and thus prevent intermolecular interactions between chitosan chains in electrospinning process [16]. Spinnability of chitosan in aqueous solutions of acetic acid also has been investigated [15, 17, 18]. Formation of nanofibers and their morphology are highly influenced by acetic acid concentration and chitosan molecular weight and percentage.

The second polymer acts as an attenuating factor to limit the hydrogen bonds between chitosan chains and thus making the electrospinning of the polymer mixture possible. For example, polyethylene oxide (PEO) $[19,20]$ and polyvinyl alcohol (PVA) [21-23] have been investigated to improve the spinnability of chitosan. They reached to the point that the formation of nanofibers is highly dependent on the mass ratio of two polymers and higher mass ratio of chitosan is just possible in lower molecular weight of chitosan. Li and Hsieh [21] showed that the maximum ratio of PVA/chitosan was just 17/83 for chitosan with medium molecular weight; therefore, they introduced alkali pretreatment to decrease the molecular weight of chitosan. This also was approved by Homayoni et al. [23]; the molecular weight of chitosan decreased with an alkali pretreatment and led to good results in increasing the chitosan ratio in the blend.

Electrospinning of $\mathrm{PCL} /$ chitosan nanofibres has only recently been performed in some mix solvent systems [19, 24-29]. Recently, PCL/chitosan nanofiber was fabricated by electrospinning of $\mathrm{PCL} /$ chitosan polymeric blend in an acetic acid/formic acid (AA/FA) solvent mixture [28]. Electrospinning of solutions containing different polymer concentration performed at the voltage which assured a stable cone. Van der Shueren et al. [28] showed that adding chitosan below $13 \mathrm{wt} \%$ PCL improved the spinnability of PCL, but there is limitation in chitosan amount as for the least possible PCL this amount is around $1.8 \mathrm{wt} \%$ and for higher amount of PCL this amount decreases significantly. Limitation of electrospinning of PCL/chitosan blend is related to the polyelectrolyte properties of chitosan which limit its solubility in acidic solutions. For 8 wt $\%$ PCL the maximum wt $\%$ chitosan is $\leq 1$. The limitations of solubility of medium molecular weight chitosan and chain entanglement problem in acidic condition affect its blend with PCL in new solvent system. So in this study alkali pretreatment of chitosan on spinnability, applicable portion of chitosan in PCL/Chitosan blend, and morphology of fabricated nanofibers were investigated.

\section{Materials and Methods}

2.1. Materials. Chitosan with medium molecular weight was purchased from Sigma-Aldrich. PCL with molecular weight $70,000-90,000$ was supplied from same company. $98 \mathrm{v} \%$ formic acid was obtained from Sigma-Aldrich, $99.8 \mathrm{v} \%$ acetic acid from Merck, and sodium hydroxide pellet from QREC (Asia) SDN BHD Malaysia.

\subsection{Methods}

2.2.1. Reducing the Molecular Weight of Chitosan. To reduce the molecular weight of chitosan, the polymer powder was treated in a $50 \% \mathrm{NaOH}$ with a chitosan/ $\mathrm{NaOH}$ ratio of $1 / 25$ $(\mathrm{w} / \mathrm{v})$ at the temperature of $95^{\circ} \mathrm{C}$ for 48 hours. Then, sample was strained and rinsed with distilled water, naturalized with acetic acid, and rinsed and dried at $60^{\circ} \mathrm{C}$ for 16 hours.

2.2.2. Preparation of the PCL/Chitosan Solutions. The electrospinning solutions were prepared by dissolving $2.5 \mathrm{wt} \%$ untreated chitosan, $7 \mathrm{wt} \%$ treated chitosan, and $8 \mathrm{wt} \% \mathrm{PCL}$ individually in the solvent mixture acetic acid/formic acid (30/70). The solutions were magnetically stirred at room temperature for three hours, time needed for complete dissolution and finally mixed with PCL/chitosan solution ratio $30 / 70,50 / 50$ and $70 / 30 \mathrm{v} / \mathrm{v} \%$ to study the effect of component ratio in electrospinning process.

2.2.3. Electrospinning. The polymer solution was pumped from a $5 \mathrm{~mL}$ syringe into a needle gauge 23 . The voltage was in the range of $18-28 \mathrm{kV}$. Electrospinning was carried out at room temperature $\left(22 \pm 2^{\circ} \mathrm{C}\right)$ and at a relative humidity of $65 \pm 5 \%$. The tip to collector distance was set at $12.5 \mathrm{~cm}$. The flow rate was set at $0.5 \mathrm{mLh}^{-1}$ and spinning was performed for $30 \mathrm{~min}$.

2.2.4. Characterization. The morphology of electrospun $\mathrm{PCL} /$ chitosan fibers was observed with a scanning electron microscope (SEM). The diameter of the fiber was measured from the SEM micrographs using image analysis software (Image J, National Institutes of Health, USA).

Viscosities of solutions were measured by Brookfield Viscometer. FTIR spectroscopy was used to identify the changes of chitosan chemical bonds after treatment and to see the presence of component in blend. Samples with same dimensions were mixed with potassium bromide $(\mathrm{KBr})$ to make pellets. FTIR spectra in the transmission mode were recorded using FTIR spectrometer (Perkin Elmer Series FTIR Spectrometer, USA), connected to a PC, and the data was analyzed by IR Solution software. 


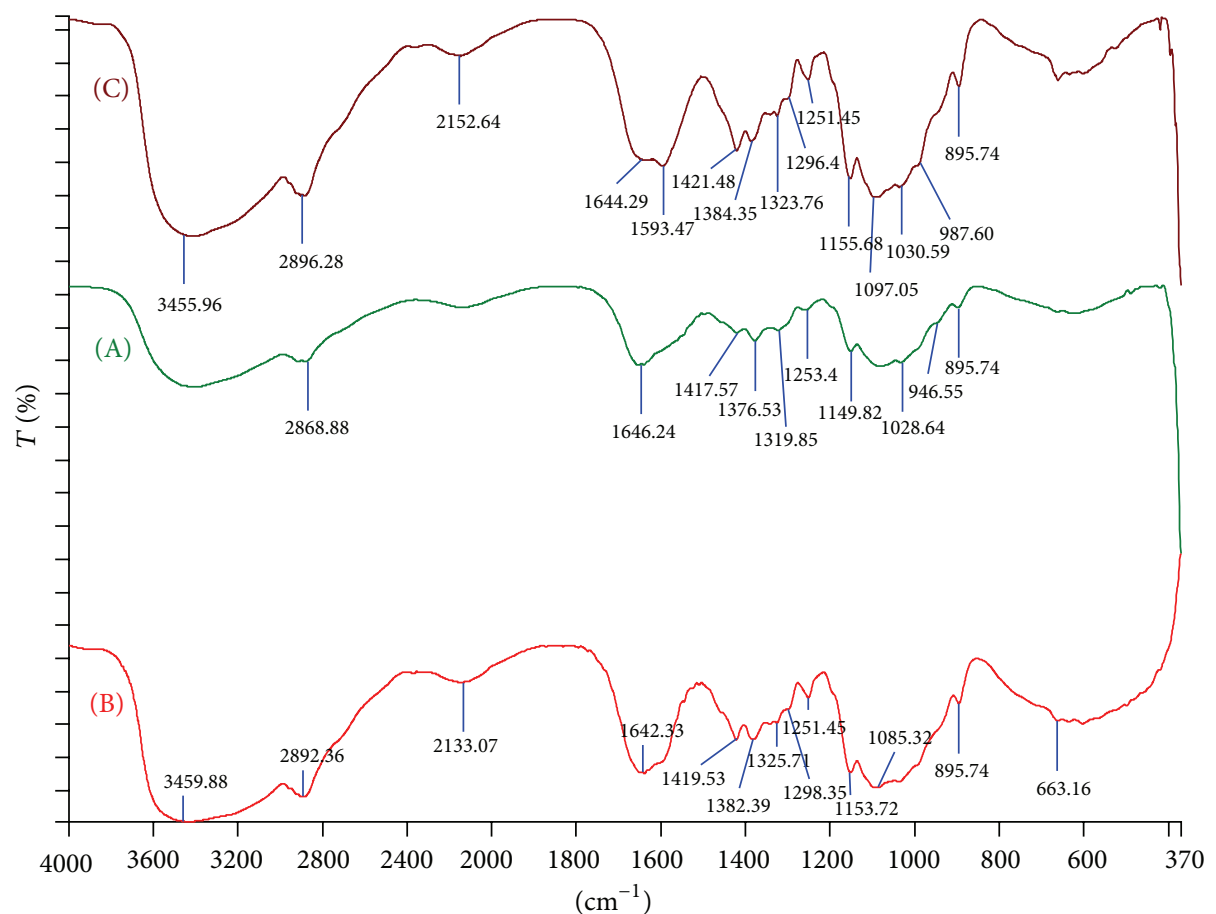

FIGURE 1: FTIR spectra of treated and untreated chitosan. (A) Untreated chitosan, (B) treated chitosan for 24 hours, and (C) treated chitosan for 48 hours.

\section{Results and Discussions}

3.1. Alkali Treatment. Alkali treatment decreases the molecular weight of chitosan by hydrolyzing the oxygen bonding the glucose amine groups. This effect on molecular weight depends on the treatment time. Chitosan treatment for 48 hours showed the best result regarding the spinnability of pure chitosan [30]. Hydrolyzing occurs more progressively by increasing treatment time. As after 5 hours molecular weight decreased from $1,094,804$ to $1,043,791$ and then by continuing the process for 16,32 , and 48 hours it changed to 645,023 , 341,782 , and finally $293,763 \mathrm{~g} / \mathrm{mol}[12]$.

Alkali treatment does not change the chemical nature of chitosan. It was proved with Fourier transform infrared (FTIR). Spectra of untreated, 24 hours, and 48 hours treated chitosan polymers are demonstrated in Figure 1. All tree spectra show the same trend at distinctive wavelengths: C$\mathrm{OH}$ groups at around $3450 \mathrm{~cm}^{-1}, \mathrm{~N}-\mathrm{H}$ groups at $1650 \mathrm{~cm}^{-1}$, and oxygen bonding at around $1150 \mathrm{~cm}^{-1}, 800 \mathrm{~cm}^{-1}$. Physical changes because of molecular weight reduction lead to some small deviations in the positions of these peaks in the spectrum of the treated chitosan corroborate.

Figure 2 shows the FTIR spectra of pure PCL and $\mathrm{PCL} /$ chitosan blend nanofibers; the distinguished feature for PCL is the carbonyl stretching absorption at around $1730 \mathrm{~cm}^{-1}$, and the spectra of PCL and chitosan blend in the form of untreated or even treated chitosan are very similar to the spectra of pure PCL except in some differences at around $3450 \mathrm{~cm}^{-1}, 1630-1670 \mathrm{~cm}^{-1}$, and $1100 \mathrm{~cm}^{-1}$ which contribute to chitosan groups as described in FTIR spectra of chitosan. In the area of $1700 \mathrm{~cm}^{-1}$, there is a deeper absorbance in
$\mathrm{PCL} /$ chitosan both treated and untreated which is related to the hydrogen bond between carbonyl groups of PCL and chitosan. So this area is identically related to interaction between PCL and chitosan.

3.2. Electrospinning. PCL/chitosan blend nanofibres provide an excellent nanostructured material for biomedical use as their valuable properties are combined into one. The new solvent system leads to production of very fine and beadless nanofibres above $13 \mathrm{wt} \%$ PCL. The electrospinning of PCL/chitosan blends using 1,1,1,3,3,3-hexafluoro-2propanol (HFIP), dimethylformamide/methylene chloride (DMF/MC), HFIP/TFA/DCM, acetone/formic acid, and acetic acid/formic acid were carried out before. Because of the toxicity and high price of most of these solvents, only the AA/FA solvent system seems applicable in PCL/chitosan electrospinning.

The main characteristic of a reproducible electrospinning process is a stable Taylor cone. Thus, voltage for all electrospinning processes has been adjusted for formation of utmost stable Taylor cone. Table 1 shows that for untreated chitosan, electrospinning of $\mathrm{PCL} /$ chitosan solution with the ratio of $70 / 30$ was performed under $21 \mathrm{kV}$ but increasing the portion of chitosan leads to polymer chain entanglement and inhibits nanofiber formation even at higher voltage. Alkali pretreatment decreases molecular weight of chitosan considerably; consequently, the viscosity of the same amounts of treated and untreated chitosan was not comparable, so for treated chitosan a solution of $7 \mathrm{wt} \%$ was prepared to make the result comparable with untreated chitosan concerning viscosities. Required voltage for polymeric blend with the ratio of $70 / 30$ 


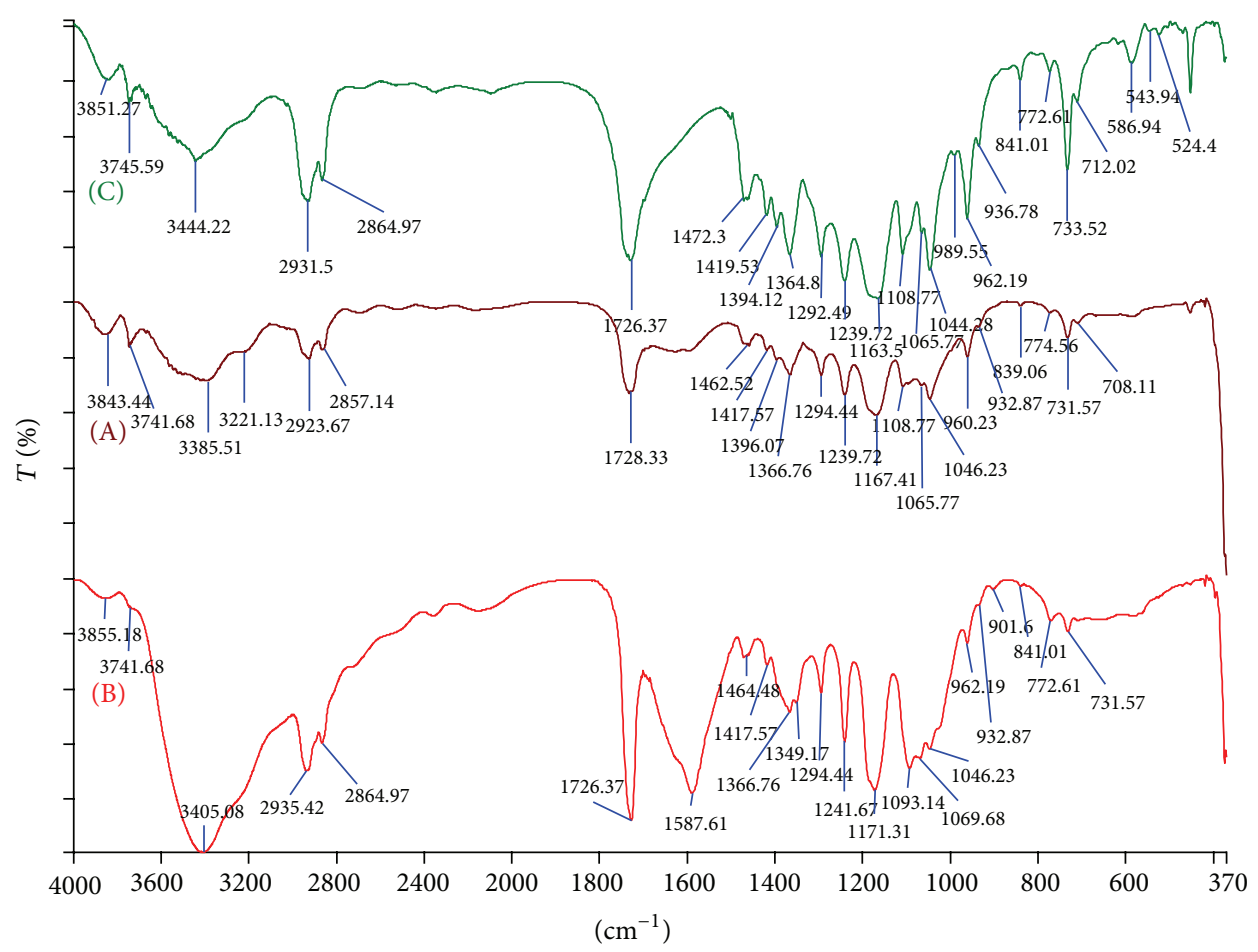

Figure 2: FTIR spectra of (A) 8 wt\% PCL/2.5\% untreated chitosan (70/30), (B) 8 wt\% PCL/7\% treated chitosan (70/30), and (C) pure PCL nanofibers.

TABLE 1: Electrospinning of PCL/chitosan blend and effect on required voltage.

\begin{tabular}{lc}
\hline PCL/chitosan blend & Applied voltage \\
\hline 8\% PCL and 2.5\% untreated chitosan (70/30) & 21 \\
8\% PCL and 7\% treated chitosan (70/30) & 25 \\
8\% PCL and 2.5\% untreated chitosan (50/50) & - \\
8\% PCL and 7\% treated chitosan (50/50) & 28 \\
\hline
\end{tabular}

$\mathrm{PCL} /$ treated chitosan was $25 \mathrm{kV}$, and for higher portion of chitosan with $50 / 50$ of $\mathrm{PCL} /$ treated chitosan a higher voltage of $28 \mathrm{kV}$ was needed.

Figures 3, 4, and 5 demonstrate the SEM images of nanofibers with the ratio of 70/30 PCL/untreated chitosan and 70/30, 50/50 PCL/treated chitosan, respectively. Table 2 shows the average diameter of nanofibers and the coefficient of variation of nanofibers. PCL/treated chitosan nanofibers with the ratio of $70 / 30$ have lower average diameter of $205 \mathrm{~nm}$ compared to $356 \mathrm{~nm}$ for blend nanofiber fabricated by untreated chitosan. Both higher applied voltage and easier movement of polymer chains are the reasons for thinner fibers for treated chitosan and PCL blend. And additionally the narrower fiber distribution is due to the higher applied voltage. Increasing the portion of chitosan in PCL/treated chitosan sample caused fabricating thinner fibers with less variation which is related to higher required voltage for spinning in a stable condition.

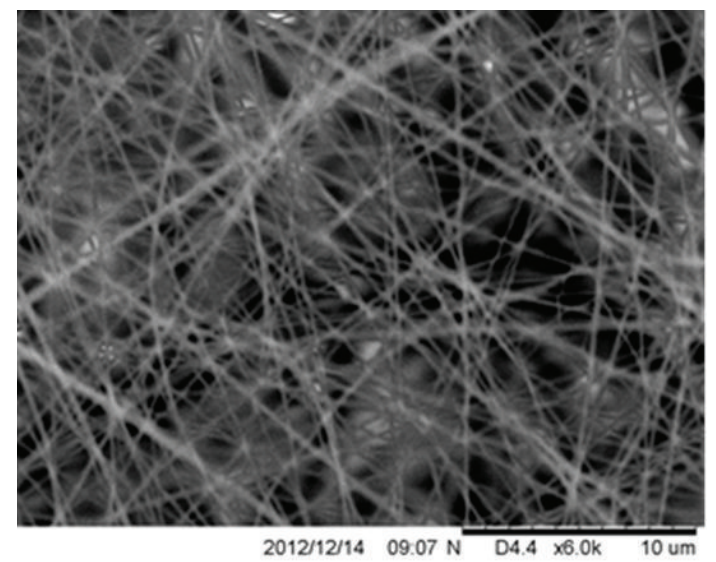

FIGURE 3: SEM image $8 \mathrm{wt} \%$ PCL/2.5\% untreated chitosan (70/30).

\section{Conclusions}

In this study, the influence of the molecular weight of chitosan on spinnability of $\mathrm{PCL} /$ chitosan blends was investigated with an electrospinning technique. Alkali treatment has been introduced as a solution to decrease the molecular weight of medium molecular weight chitosan and, thus, reduction of hindering effect of positively charged amine group when spinning. This treatment does not change the chemical structure of chitosan. Treated chitosan in blend with PCL nanofibers caused fabrication of smaller diameter and narrower deviation compared to untreated chitosan. Smaller 


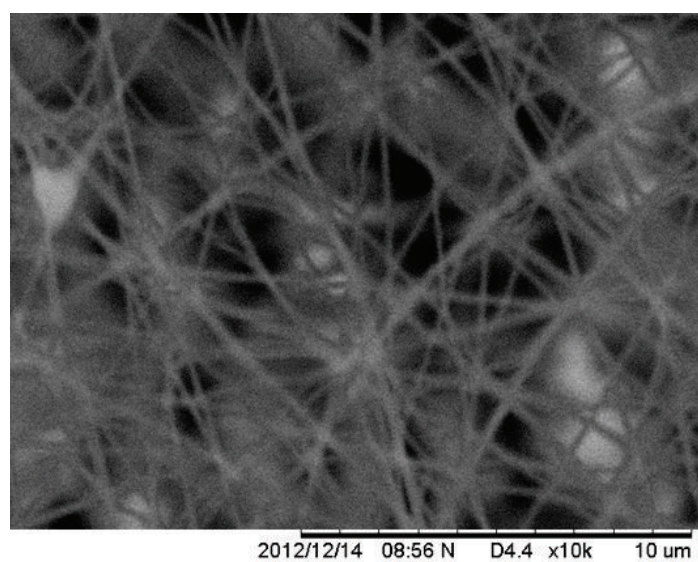

Figure 4: SEM image 8 wt\% PCL/7\% treated chitosan (70/30).

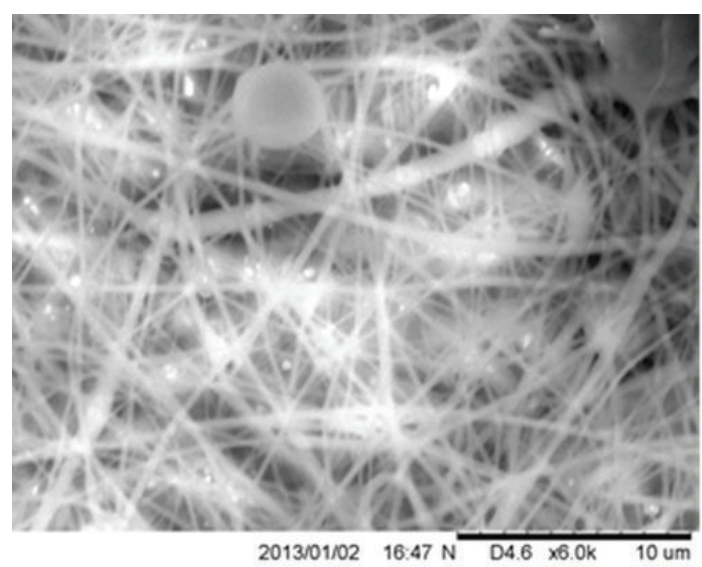

FIGURE 5: SEM image 8 wt\% PCL/7\% alkali treated chitosan (50/50).

TABLE 2: Average diameter, coefficient of variation, and surface porosity of chitosan blend.

\begin{tabular}{lcc}
\hline PCL/chitosan blend & $\begin{array}{c}\text { Average diameter } \\
\text { (nm) }\end{array}$ & $\begin{array}{c}\text { Coefficient of } \\
\text { variation \% }\end{array}$ \\
\hline $\begin{array}{l}\text { 8\% PCL and 2.5\% untreated } \\
\text { chitosan (70/30) }\end{array}$ & 356.29 & 172.10 \\
$\begin{array}{l}\text { 8\% PCL and 7\% treated } \\
\text { chitosan (70/30) }\end{array}$ & 204.81 & 83.92 \\
$\begin{array}{l}\text { 8\% PCL and 2.5\% untreated } \\
\text { chitosan (50/50) }\end{array}$ & - & - \\
$\begin{array}{l}\text { 8\% PCL and 7\% treated } \\
\text { chitosan (50/50) }\end{array}$ & 317.46 & 67.37 \\
\hline
\end{tabular}

diameter is related to easier movement of chitosan polymer chains exposed to high voltage during electrospinning. Also increasing chitosan ratio in blend with PCL is possible to be considered for further studies in biomedical application.

\section{Acknowledgments}

F. Roozbahani would like to thank the University Teknologi Malaysia for International Doctoral Fellowship. The authors also acknowledge MOHE, GUP Tier 1 Grants (Vot: 03H13, Vot: 05H07), FRGS (Vot: 4F126), MOHE, UTM, and RMC for financial support. Lab facilities of FBME are also acknowledged.

\section{References}

[1] D. F. Stamatialis, B. J. Papenburg, M. Gironés et al., "Medical applications of membranes: drug delivery, artificial organs and tissue engineering," Journal of Membrane Science, vol. 308, no. 1-2, pp. 1-34, 2008.

[2] N. Sultana and M. Wang, "PHBV/PLLA-based composite scaffolds fabricated using an emulsion freezing/freeze-drying technique for bone tissue engineering: surface modification and in vitro biological evaluation," Biofabrication, vol. 4, no. 1, Article ID 015003, 2012.

[3] N. Sultana and T. H. Khan, "In vitro degradation of PHBV scaffolds and nHA/PHBV composite scaffolds containing hydroxyapatite nanoparticles for bone tissue engineering," Journal of Nanomaterials, vol. 2012, Article ID 190950, 12 pages, 2012.

[4] I. Armentano, M. Dottori, E. Fortunati, S. Mattioli, and J. M. Kenny, "Biodegradable polymer matrix nanocomposites for tissue engineering: a review," Polymer Degradation and Stability, vol. 95, no. 11, pp. 2126-2146, 2010.

[5] R. Ravichandran, S. Sundarrajan, J. R. Venugopal, S. Mukherjee, and S. Ramakrishna, "Advances in polymeric systems for tissue engineering and biomedical applications," Macromolecular Bioscience, vol. 12, no. 3, pp. 286-311, 2012.

[6] J. Dobkowski, R. Kołos, J. Kamiński, and H. M. Kowalczyńska, "Cell adhesion to polymeric surfaces: experimental study and simple theoretical approach," Journal of Biomedical Materials Research, vol. 47, no. 2, pp. 234-242, 1999.

[7] H. Yoshimoto, Y. M. Shin, H. Terai, and J. P. Vacanti, "A biodegradable nanofiber scaffold by electrospinning and its potential for bone tissue engineering," Biomaterials, vol. 24, no. 12, pp. 2077-2082, 2003.

[8] C. M. Vaz, S. van Tuijl, C. V. C. Bouten, and F. P. T. Baaijens, "Design of scaffolds for blood vessel tissue engineering using a multi-layering electrospinning technique," Acta Biomaterialia, vol. 1 , no. 5, pp. 575-582, 2005 .

[9] F. Chen, C. N. Lee, and S. H. Teoh, "Nanofibrous modification on ultra-thin poly(e-caprolactone) membrane via electrospinning," Materials Science and Engineering C, vol. 27, no. 2, pp. 325-332, 2007.

[10] S.-C. Wong, A. Baji, and S. Leng, "Effect of fiber diameter on tensile properties of electrospun poly(E-caprolactone)," Polymer, vol. 49, no. 21, pp. 4713-4722, 2008.

[11] K. Ohkawa, H. Kim, K. Lee, and H. Yamamoto, "Electrospun non-woven fabrics of poly ( $\epsilon$-caprolactone) and their biodegradation by pure cultures of soil filamentous fungi," Macromolecular Symposia, vol. 216, no. 1, pp. 301-306, 2004.

[12] A. K. Moghe, R. Hufenus, S. M. Hudson, and B. S. Gupta, "Effect of the addition of a fugitive salt on electrospinnability of poly $(\varepsilon-$ caprolactone)," Polymer, vol. 50, no. 14, pp. 3311-3318, 2009.

[13] L. van der Schueren, B. de Schoenmaker, Ö. I. Kalaoglu, and K. de Clerck, "An alternative solvent system for the steady state electrospinning of polycaprolactone," European Polymer Journal, vol. 47, no. 6, pp. 1256-1263, 2011.

[14] C. Mahoney, M. B. McCullough, J. Sankar, and N. Bhattarai, "Nanofibrous structure of chitosan for biomedical applications," Journal of Nanomedicine and Biotherapeutic Discovery, vol. 2, no. $1,2012$. 
[15] K. Ohkawa, D. Cha, H. Kim, A. Nishida, and H. Yamamoto, "Electrospinning of chitosan," Macromolecular Rapid Communications, vol. 25, no. 18, pp. 1600-1605, 2004.

[16] K. Sun and Z. H. Li, "Preparations, properties and applications of chitosan based nanofibers fabricated by electrospinning," Express Polymer Letters, vol. 5, no. 4, pp. 342-361, 2011.

[17] X. Geng, O.-H. Kwon, and J. Jang, "Electrospinning of chitosan dissolved in concentrated acetic acid solution," Biomaterials, vol. 26, no. 27, pp. 5427-5432, 2005.

[18] S. de Vrieze, P. Westbroek, T. van Camp, and L. van Langenhove, "Electrospinning of chitosan nanofibrous structures: feasibility study," Journal of Materials Science, vol. 42, no. 19, pp. 80298034, 2007.

[19] N. Bhattarai, Z. Li, J. Gunn et al., "Natural-synthetic polyblend nanofibers for biomedical applications," Advanced Materials, vol. 21, no. 27, pp. 2792-2797, 2009.

[20] K. Ziani, C. Henrist, C. Jérôme, A. Aqil, J. I. Maté, and R. Cloots, "Effect of nonionic surfactant and acidity on chitosan nanofibers with different molecular weights," Carbohydrate Polymers, vol. 83, no. 2, pp. 470-476, 2011.

[21] L. Li and Y.-L. Hsieh, "Chitosan bicomponent nanofibers and nanoporous fibers," Carbohydrate Research, vol. 341, no. 3, pp. 374-381, 2006.

[22] M. Ignatova, K. Starbova, N. Markova, N. Manolova, and I. Rashkov, "Electrospun nano-fibre mats with antibacterial properties from quaternised chitosan and poly(vinyl alcohol)," Carbohydrate Research, vol. 341, no. 12, pp. 2098-2107, 2006.

[23] H. Homayoni, S. A. H. Ravandi, and M. Valizadeh, "Influence of the molecular weight of chitosan on the spinnability of chitosan/poly(vinyl alcohol) blend nanofibers," Journal of Applied Polymer Science, vol. 113, no. 4, pp. 2507-2513, 2009.

[24] A. Cooper, N. Bhattarai, and M. Zhang, "Fabrication and cellular compatibility of aligned chitosan-PCL fibers for nerve tissue regeneration," Carbohydrate Polymers, vol. 85, no. 1, pp. 149-156, 2011.

[25] S. Hong and G. Kim, "Fabrication of electrospun polycaprolactone biocomposites reinforced with chitosan for the proliferation of mesenchymal stem cells," Carbohydrate Polymers, vol. 83, no. 2, pp. 940-946, 2011.

[26] M. P. Prabhakaran, J. R. Venugopal, T. T. Chyan et al., "Electrospun biocomposite nanofibrous scaffolds for neural tissue engineering," Tissue Engineering A, vol. 14, no. 11, pp. 1787-1797, 2008.

[27] K. T. Shalumon, K. H. Anulekha, C. M. Girish, R. Prasanth, S. V. Nair, and R. Jayakumar, "Single step electrospinning of chitosan/poly(caprolactone) nanofibers using formic acid/acetone solvent mixture," Carbohydrate Polymers, vol. 80, no. 2, pp. 414420, 2010.

[28] L. van der Schueren, I. Steyaert, B. de Schoenmaker, and K. de Clerck, "Polycaprolactone/chitosan blend nanofibres electrospun from an acetic acid/formic acid solvent system," Carbohydrate Polymers, vol. 88, no. 4, pp. 1221-1226, 2012.

[29] X. Yang, X. Chen, and H. Wang, "Acceleration of osteogenic differentiation of preosteoblastic cells by chitosan containing nanofibrous scaffolds," Biomacromolecules, vol. 10, no. 10, pp. 2772-2778, 2009.

[30] H. Homayoni, S. A. H. Ravandi, and M. Valizadeh, "Electrospinning of chitosan nanofibers: processing optimization," Carbohydrate Polymers, vol. 77, no. 3, pp. 656-661, 2009. 

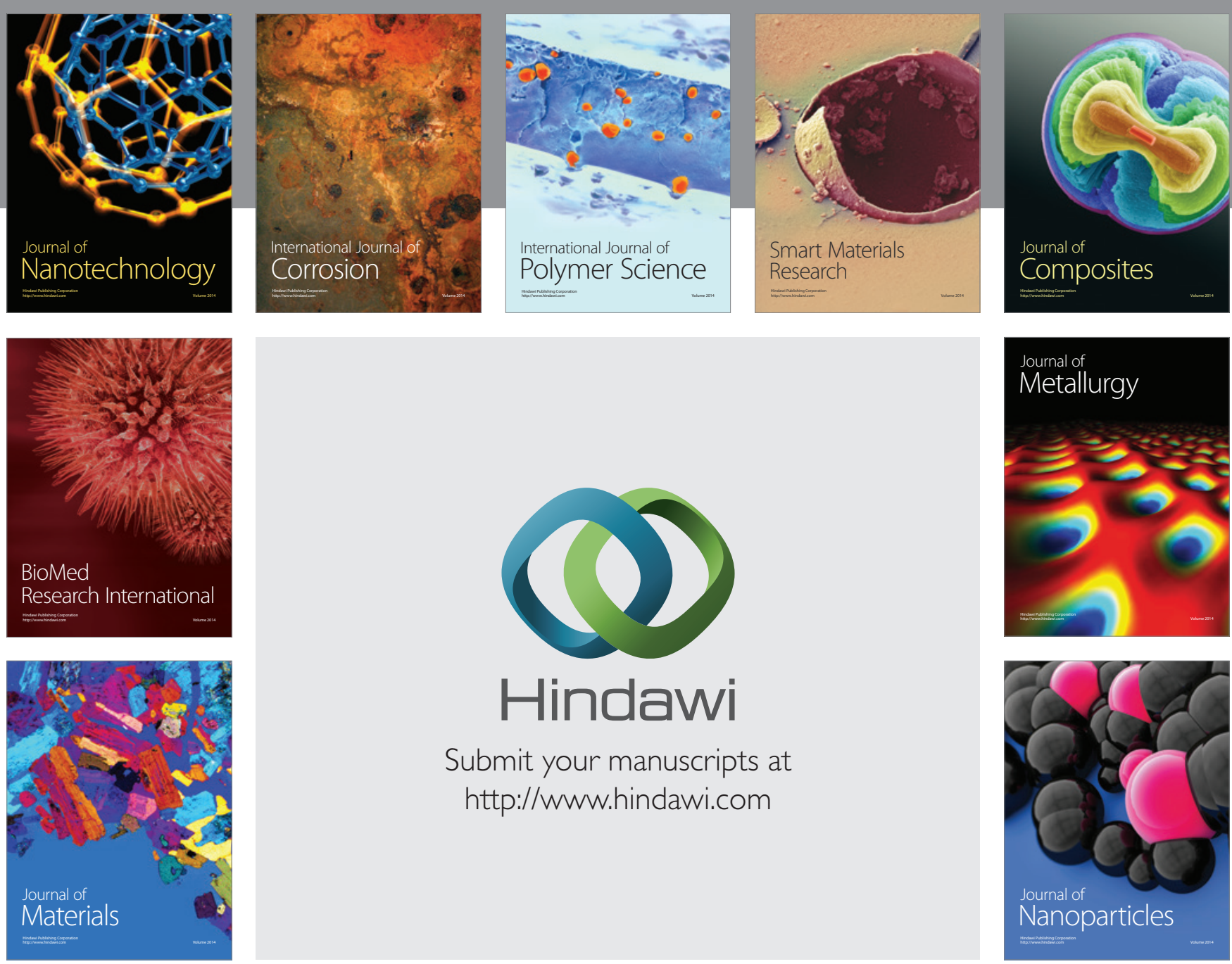

Submit your manuscripts at http://www.hindawi.com
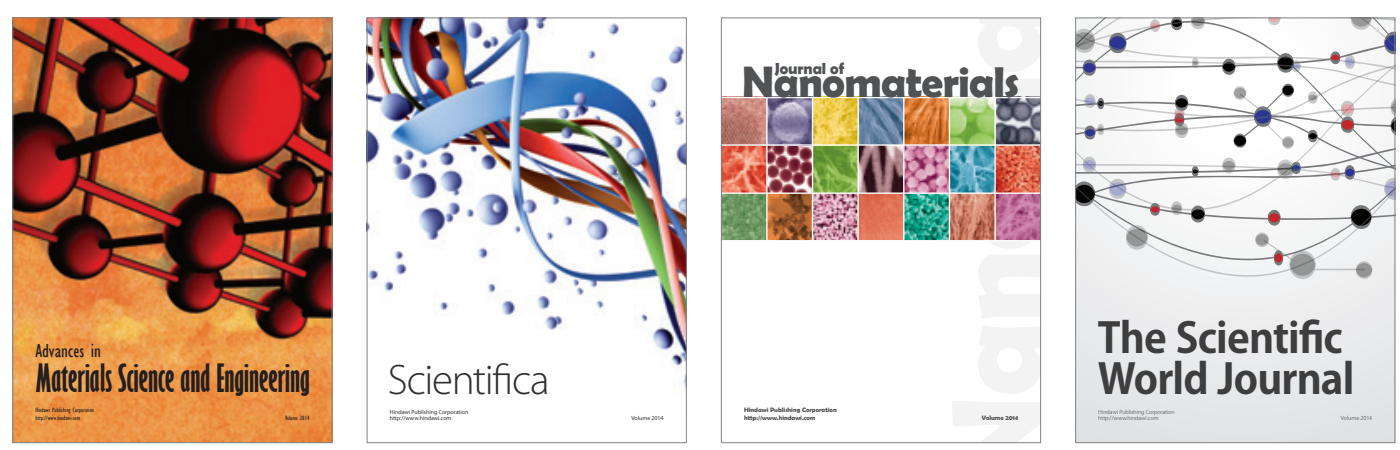

\section{The Scientific World Journal}
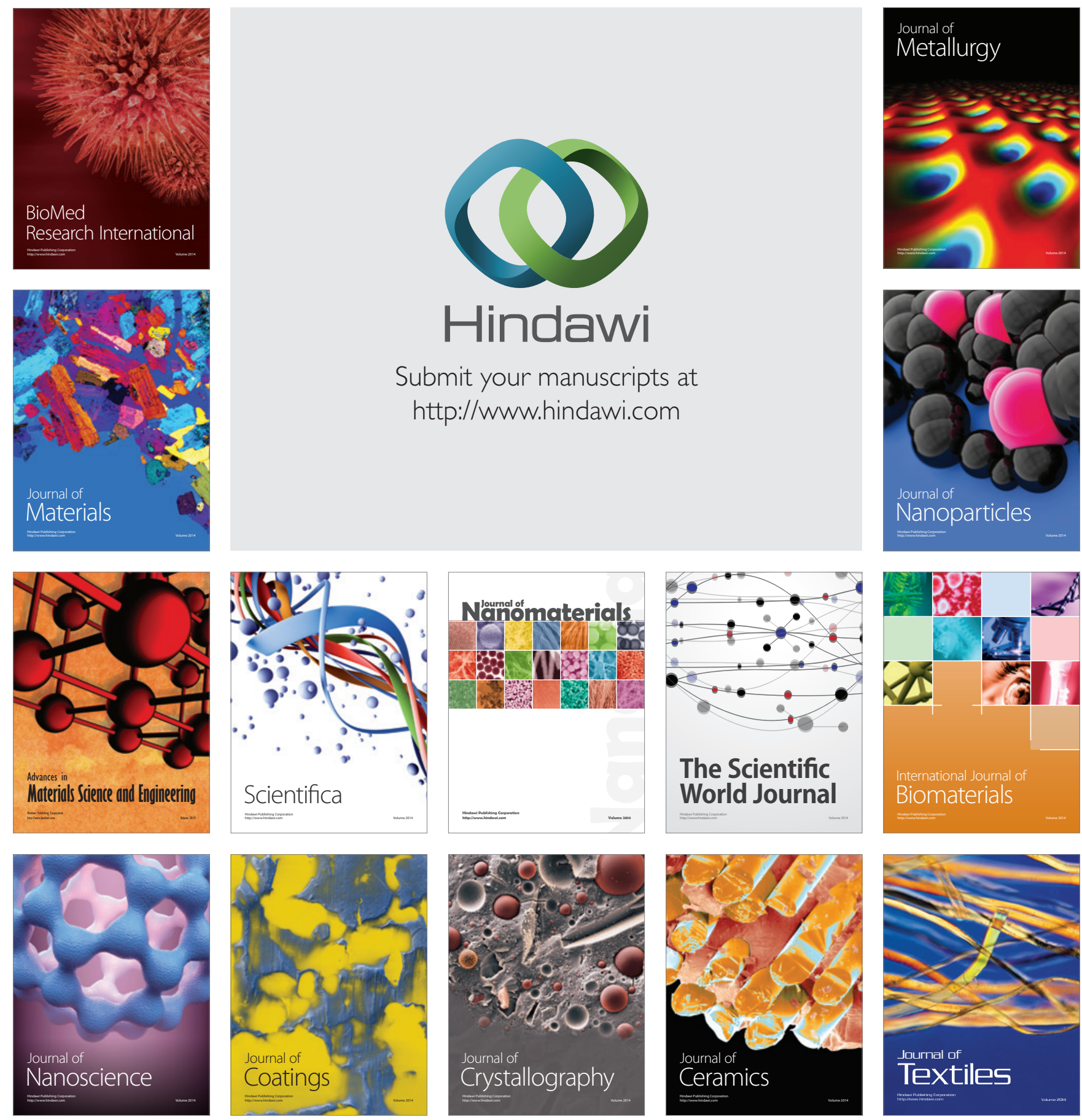